\title{
Spectroscopy of southern $\delta$ Scuti stars
}

\author{
Malcolm Cropp, Karen R. Pollard, Jovan Skuljan \\ Department of Physics and Astronomy, University of Canterbury, \\ Private Bag 4800, Christchurch, New Zealand
}

\begin{abstract}
Four $\delta$ Scuti stars were observed with the HERCULES fibrefed échelle spectrograph at Mount John University Observatory, New Zealand. These observations were analysed by looking at the radial velocity variations as given by a cross-correlation technique as well as spectral line moment variations. These results were compared to published photometric studies of these stars to see if the modes identified in the photometry were also present in the spectroscopic data obtained.
\end{abstract}

\section{Introduction and observations}

The pulsations in a sample of southern $\delta$ Scuti stars were investigated by studying radial velocity and line profile variations. Two different methods of analysis were employed (the radial velocity cross correlation technique and the line moment analysis) to examine if either method could identify periodicities in the data and recover known (generally) photometric periods. As the pulsations in these stars are non-radial with small radial components, periodicities in the line profile variations are likely to be more evident. At least one star in our sample is a known spectroscopic binary (HD $3112=\theta$ Tuc) so the radial velocity variations due to the orbital motion should be readily identified.

All observations were obtained with the 1-m McLellan telescope at Mount John University Observatory (MJUO). The instrument used was the HERCULES spectrograph with a Photometrics Series 200 CCD camera system incorporating a SITe 003AB CCD chip. Time series spectroscopic observations were made of the target stars as well as a number of stars used as radial velocity standards. Table 1 presents a list of the programme stars observed.

Table 1. $\delta$ Scuti stars observed at MJUO, with information on their $V$ magnitude, period, spectral type and number of observations.

\begin{tabular}{|c|c|c|c|c|c|c|c|}
\hline \multirow[b]{2}{*}{ Star } & \multirow[b]{2}{*}{ HD } & \multicolumn{2}{|c|}{2000.0 coordinates } & \multirow[b]{2}{*}{$V$} & \multirow{2}{*}{$\begin{array}{l}\text { Period } \\
\text { (hr) }\end{array}$} & \multirow{2}{*}{$\begin{array}{c}\text { Spectral } \\
\text { type }\end{array}$} & \multirow{2}{*}{$\begin{array}{c}\text { Total } \\
\text { obs }\end{array}$} \\
\hline & & RA & Dec & & & & \\
\hline$\theta$ Tuc & 3112 & 003323 & -7116.0 & 6.11 & 1.18 & A7IV & 187 \\
\hline BN Hyi & 20313 & 030732 & -7859.4 & 5.67 & 1.58 & F2III & 203 \\
\hline FZ Vel & 77140 & 085852 & -4714.1 & 5.17 & 1.56 & $\mathrm{Am}$ & 190 \\
\hline FG Vir & 106384 & 121415 & -0543.0 & 6.56 & 1.89 & A5 & 109 \\
\hline
\end{tabular}




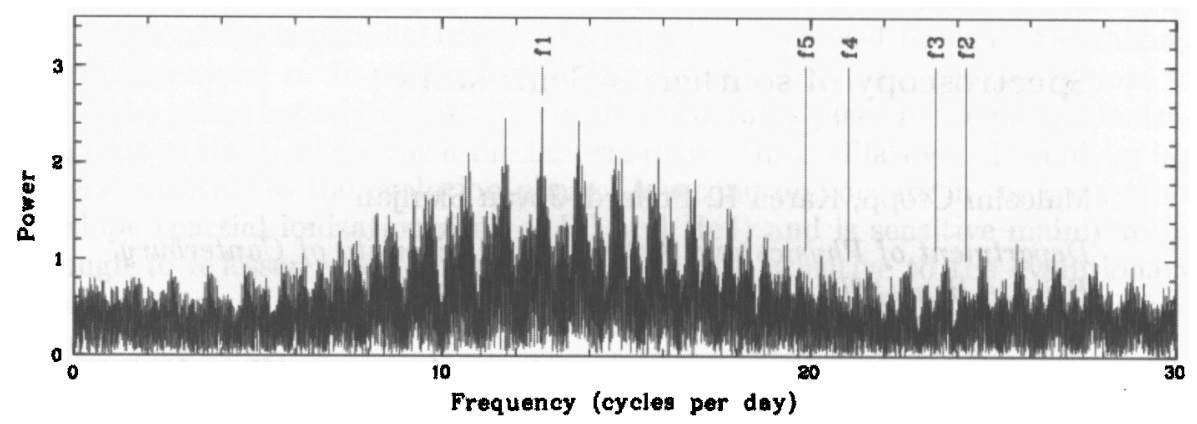

Figure 1. The power spectrum for the HD 106384 radial velocity data. The first five frequencies found by Breger et al. (1999) are indicated on the figure. These frequencies are $f_{1}=12.716 \mathrm{~d}^{-1}$, $f_{2}=24.228 \mathrm{~d}^{-1}, f_{3}=23.402 \mathrm{~d}^{-1}, f_{4}=21.052 \mathrm{~d}^{-1}$ and $f_{5}=19.868 \mathrm{~d}^{-1}$.

\section{Results}

Each programme $\delta$ Scuti star was analysed by a cross correlation method to produce a radial velocity shift with respect to a high signal-to-noise reference frame of that star. The resulting radial velocities were then examined for periodic behaviour using standard Fourier techniques. The periodogram for HD 106384 is shown in Fig. 1.

The frequencies found for HD 3112 were $0.1309 \pm 0.0003 \mathrm{~d}^{-1}$ and $19.022 \pm$ $0.002 \mathrm{~d}^{-1}$. HD 3112 is a binary system with the primary being a $\delta$ Scuti variable (De Mey et al. 1998) and the low frequency detected is due to the orbital motion. The second frequency found is believed to be due to a mode with radial degree $\ell=1$ (Paparo et al. 1996).

The radial velocity data for HD 20313 showed a strong frequency at $0.1180 \pm$ $0.0001 \mathrm{~d}^{-1}$, but no other significant frequencies were detected in the spectroscopic data set. This suggests that this star, like HD 3112, is a spectroscopic binary candidate. Fig. 2 shows a fit of this (likely-orbital) period to the radial velocity data obtained at MJUO.

The results for HD 106384 showed two easily-detectable frequencies in the radial velocity data. These are $12.7163 \pm 0.0003 \mathrm{~d}^{-1}$ and $24.690 \pm 0.002 \mathrm{~d}^{-1}$. These correspond very well to the first two frequencies detected in extensive photometric studies of the star (Breger et al. 1999). In addition to studying the radial velocities of this star, a technique was used to investigate the first moment of a spectral line (as described in Balona 1986). This revealed the $12.716 \mathrm{~d}^{-1}$ frequency as well as an additional frequency of $21.210 \pm 0.002 \mathrm{~d}^{-1}$ which was also detected by Breger et al. (1999).

The radial velocity data for HD 77140 did not show any significant periods. This indicates that any pulsational modes have very small radial components that our current spectroscopic dataset is unable to uncover.

A summary of the periods found for each programme $\delta$ Scuti star by the radial velocity cross correlation and moment techniques is shown in Table 2 . 


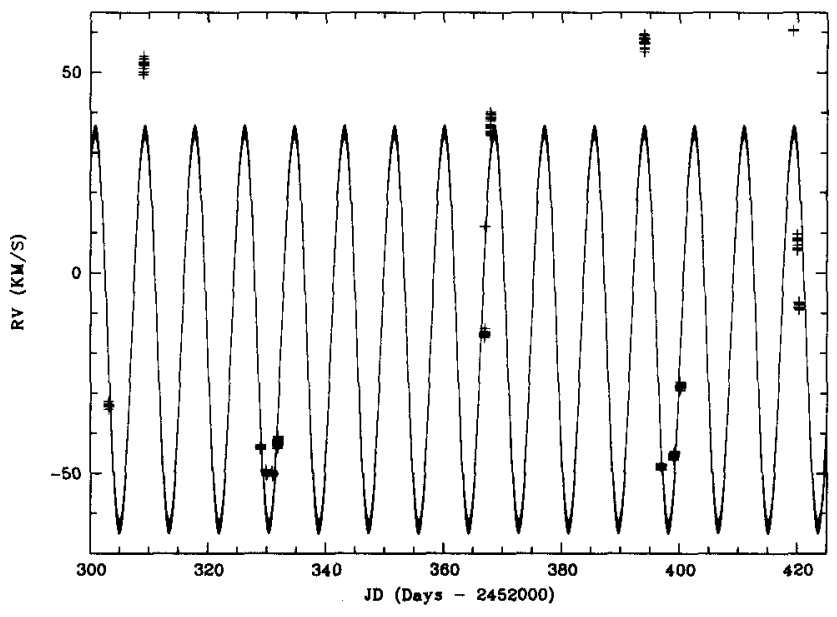

Figure 2. The observed radial velocities for HD 20313 are plotted along with the fit calculated for a frequency of $0.1180 \mathrm{~d}^{-1}$ (the suggested orbital period of the spectroscopic binary system). The rms scatter in the fit is $15 \%$ of the peak-to-peak amplitude.

\section{Conclusion}

The use of spectroscopy to detect the dominant periodicities in these southern $\delta$ Scuti stars was successful in three out of the four stars studied. For HD 106384, three frequencies were found. These matched well with frequencies found in previous photometric studies. Analysis of HD 3112 revealed two frequencies which matched with photometric results, and for HD 20313 one frequency was uncovered which is most likely to be the orbital period of the spectroscopic binary system. For HD 77140 no significant frequencies were found in the spectroscopy.

Table 2. A summary of the periods found for each programme $\delta$ Scuti star by the radial velocity cross correlation and moment techniques.

\begin{tabular}{clcc}
\hline Star & $\begin{array}{l}\text { Frequencies found in } \\
\text { radial velocity }\left(\mathrm{d}^{-1}\right)\end{array}$ & $\begin{array}{c}\text { Frequencies found in } \\
\text { line moments }\left(\mathrm{d}^{-1}\right)\end{array}$ & $\begin{array}{c}\text { Radial degree } \\
(\ell)\end{array}$ \\
\hline HD 3112 & $f_{1}=0.1309 \pm 0.0003$ & & \\
& $f_{5}=19.022 \pm 0.002$ & & 1 \\
HD 20313 & $f_{1}=0.118 \pm 0.004$ & $f_{1}=15.726 \pm 0.001$ & \\
HD 77140 & & $f_{1}=12.7158 \pm 0.0004$ & 1 \\
HD 106384 & $f_{1}=12.7163 \pm 0.0003$ & 1 \\
& $f_{2}=24.690 \pm 0.002$ & $f_{4}=21.210 \pm 0.002$ & 2 \\
\hline
\end{tabular}




\section{References}

Balona, L. 1986, MNRAS, 219, 111

Breger, M., Pamyatnykh, A., Pikall, H., Garrido, R. 1999, A\&A, 341, 151

De Mey, K., Daems, K., Sterken, C. 1998, A\&A, 336, 527

Paparo, M., Sterken, C., Spoon, H., Birch, P. 2001, A\&A, 366, 178

Rodriguez, E., Lopez-Gonzalez, M., Lopez de Coca, P. 2000, A\&AS, 144, 469

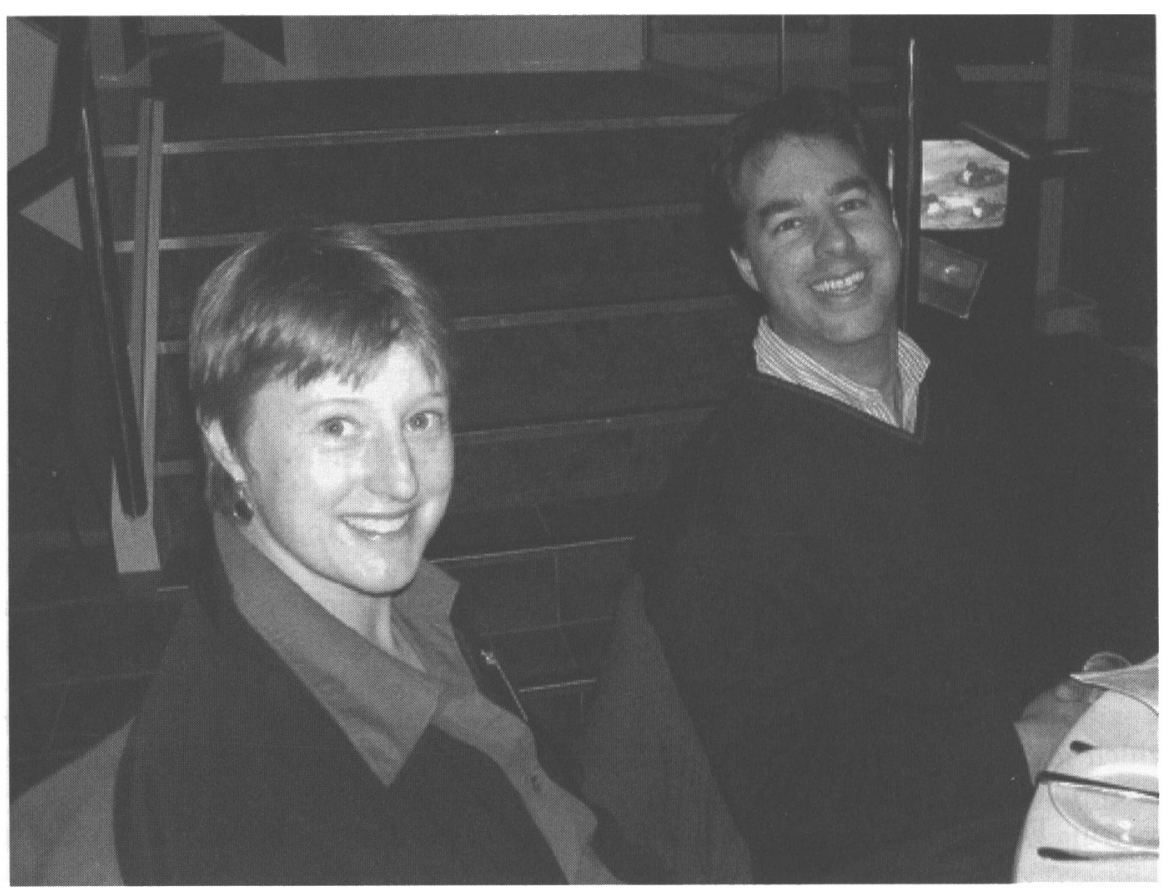

Karen Pollard and Michael Albrow 